\title{
The "Dimming Effect" Produced by the Application of Doppler Effect on the Quantity of Photons Arriving to a Receiver and its Implication to Astronomy
}

\author{
M. Zilberman, M.Sc.* \\ Independent researcher, Canada
}

\begin{abstract}
This article describes the dimming effect that is produced by the Doppler effect applied to a quantity of individual photons arriving to a receiver from a moving source of light. The corpuscular-wave dualism of light suggests that the well-known Doppler effect, which is currently applied only to the wave component of light, should also be considered for the corpuscular component of light. Application of the Doppler effect on a quantity of photons leads to the "Dimming Effect" - as the faster light source is moving away from observer - the dimmer its brightness appears. While the described dimming effect is negligible for low-speed light sources, it becomes significant for light sources with a velocity comparable to light speed in a vacuum. The relativistic adjustments for time dilation cause the described dimming effect to be even stronger. For example, the "Dimming Effect" for an object moving away from the observer with the speed $0.1 c$ is 0.904 and for an object moving away from the observer with the speed $0.5 c$ is 0.577 . If confirmed, the dimming effect must be taken into account in calculations of astronomical "Standard Candles" and in particular in the "Supernova Cosmology Project", which has claimed the acceleration of the Universe's expansion and led to the introduction of dark energy.
\end{abstract}

Keywords: Doppler effect, "Standard Candles", "Supernova Cosmology Project”, dark energy, dimming

\section{The Doppler effect for waves}

The Doppler effect is well known [1], [2] and widely used in science and engineering. This formula (1) describes the change of light frequency in a vacuum due to the Doppler effect.

$$
F=F_{0} \cdot \frac{\sqrt{1-\frac{v^{2}}{c^{2}}}}{1-\frac{v}{c} \cdot \cos \theta}
$$

Here $F_{0}$ is the radiation frequency at the source of light, $F$ is the observed radiation frequency, $C$ is the speed of light, $v$ is the speed of the source relative to the observer and $\theta$ is the angle between the direction to the source and the velocity vector in the receiver's frame of reference. If the source is radially away from the observer, then $\theta=\pi$, if it approaches, then $\theta=0$.

In astronomy the Doppler effect particularly allows the estimation of distance to far galaxies using the correspondence between their distance and redshift of the galaxies spectra.

\footnotetext{
*email address: MZilberman137@gmail.com
} 
On the other hand, the corpuscular-wave dualism of light is also well known. Currently the attention of physicists was focused to the application of the Doppler effect on the wave component of light. This likely occurred because the Doppler effect on sound (the entire wave object) was well researched. Upon a scan of the published literature on the topic, there do not appear to be any articles that analyzed the application of the Doppler effect on the corpuscular component of light (including relativistic effects).

\section{Thought experiment}

Imagine we watch a movie where a police car chases criminals. Since we are only concerned in the relative speed of objects, we can imagine that a police car is static and the criminal's car is distancing from it with the fixed relative speed $V$ (meters per second). For simplicity we consider an one-dimensional model and neglect any air resistance. During every second, the criminal in the car shoots one bullet at the police car. Let's denote the speed of the bullet as $S$ (meters per second) and the initial distance between cars as $D$ (meters). We also assume that $S \gg V$, not to make calculations more complex by subtracting the speed of car from the speed of bullet.

The bullet that was shot on the second number " 0 " will reach the police car after the $D / S$ seconds on the moment $D / S$ (all calculations done in the police car reference frame). During the period between the first and second shot the criminal's car moves $V(\mathrm{~m} / \mathrm{s}) \times(1$ second $)=V$ meters. Therefore, the second shot will be shot from the distance $D+V$ meters. The second bullet will hit the police car after $(D+V) / S$ seconds during the moment $(1$ second $)+(D+V) / S$. (The moment "1 second" is the moment when it was shot plus the bullet's flying time).

During the time between the second and third shots the criminal's car moves an additional $V$ meters. Therefore, the third shot will be fired from the distance of $D+V^{* 2}$ meters. The third bullet will hit the police car after $\left(D+V^{* 2}\right) / S$ seconds during the moment $(2$ seconds $)+$ $\left(D+V^{* 2}\right) / S$. (The moment " 2 seconds" is the moment when the bullet was shot plus the bullet's flying time). And so forth.

Next, let's calculate the time interval between sequential bullets hitting the police car. The first bullet hits the police car during moment $D / S$. The second bullet hits the police car during the moment $(1$ second $)+(D+V) / S$. Therefore, the time interval between sequential bullets hitting are

$$
d T=1+(D+V) / S-D / S=1+D / S+V / S-D / S=1+V / S
$$

This result is almost obvious. If the criminal car does not drive (i.e. $V=0$ ) or if the bullets have infinite speed $(S \rightarrow \infty)$ then the bullets hit the car every second. If the speed of the criminal car is not 0 and the speed of bullets is not infinite, then the frequency of strikes is described by the formula (2). Let's also note that if the criminal's car is approaching the police car (i.e. $V<0)$ the interval between bullet strikes will be less than 1 second, which is also quite obvious.

From the formula (2) the number of bullets that strike the police car during the time interval $\mathrm{T}$ (seconds) is calculated as 


$$
N=\frac{T}{1+\frac{V}{S}}
$$

Since our model assumed a 1-dimensional movement of the car and does not consider any relativistic effects it can be understood that formula (3) and the classical formula (1) both describe the Doppler effect. The difference however is in the nature of objects they describe. Formula (1) describes the wave object (sound, light, etc.) and formula (3) describes the movement of discrete objects.

Since the number of shots during the interval $\mathrm{T}$ in seconds is also $\mathrm{T}$ (one shot per second) the number of bullets shot $\left(\mathrm{N}_{0}\right)$ and the number of bullets received $(\mathrm{N})$ per second is given by the formula (4). Depending on the direction of criminal's car, the value $\mathrm{N}$ can be less than $\mathrm{N}_{0}$ (criminal's car is moving away) or greater than $\mathrm{N}_{0}$ (criminal's car is approaching.)

$$
N=\frac{N_{0}}{1+\frac{V}{S}}
$$

\section{Application to the photon model of light}

Now let's consider the corpuscular-wave nature of light and apply the obtained formula (4) to the numbers of emitted and received photons. Let's consider an object that emits photons. If this object is not approaching the observer and is not moving away from the observer $(V=0)$, then the numbers of emitted and received photons per second are the same. However, if the object is moving to or from the observer, these numbers become different and are described by formula (4). If the source and receiver of photons are located in a vacuum, then formula (4) appears as

$$
N=\frac{N_{0}}{1+\frac{V}{C}}
$$

Here $c$ is the speed of light in a vacuum, $V$ is the speed, with which object that emits photons is moving away from observer, $\mathrm{N}_{0}$ is the number of emitted photons and $\mathrm{N}$ is the number of observed photons during a fixed time interval.

Since we define brightness of objects by the number of photons arriving to a receiver during a predefined time interval, in accordance to formula (5), the light sources approaching us $(V<0)$ will look brighter and the light sources moving away $(V>0)$ from us will look dimmer.

However, since in real life the speed of the light sources $V$ is much less than $c$, we do not observe this effect. For example, if 2 observers located at diametrically opposite points of the earth's 
equator attempt to measure the brightness of a distant object of light, located in the equatorial plane, the difference in brightness will only be

$$
460(\mathrm{~m} / \mathrm{s}) * 2 / 300,000,000(\mathrm{~m} / \mathrm{s})=3.06667 \mathrm{E}-06,
$$

where $460 \mathrm{~m} / \mathrm{s}$ is the Earth's rotating speed at the equator.

Optimistically, there is a chance that physicists can offer further sophisticated methods of affirming formula (5).

\section{Application to astronomy}

In astronomy, however, formula (5) can be tested. If we observe 2 similar objects and know their speeds and distances, we will be able to tell if their brightness depends on their speed or not. Since astronomy deals with certain objects whose speed is comparable to the speed of light in a vacuum these measurements appear attainable. For example, for an object moving away from us with the speed of $0.1 c(30,000 \mathrm{~km} / \mathrm{s})$ the effect of dimming is

$$
\mathrm{N} / \mathrm{N}_{0}=\frac{1}{1+0.1}=0.91
$$

For the object moving away from us with the speed $0.5 c(150,000 \mathrm{~km} / \mathrm{s})$ the effect of dimming is

$$
N / N_{0}=\frac{1}{1+0.5}=0.66
$$

Two comments.

a. All the above calculations do not yet take into account relativistic effects.

b. The assumption $S \gg V$ from the "thought experiment" above is not applicable to formulas (5) and (6) because the $S$ and $V$ values are now comparable. However, since we consider the emission of photons, the speed $S$ is always equal to $c$ and therefore is independent from the speed $V$ of the light source. (In the "thought experiment" above we used the $S \gg V$ assumption not to make calculations more complex by subtracting the speed of car from the speed of bullet. For emission of photons situation is different since their speed in vacuum is always equal to $c$.)

\section{Relativistic effects}

Since we consider the movement of objects with speed comparable to $c$, we must account for their relativistic effects and relativistic time dilation. According to Special Relativity the processes in the moving inertial reference frame will appear slower with the coefficient $\sqrt{1-\frac{v^{2}}{c^{2}}}$, where $v$ is the speed of the object and $c$ is the speed of light in a vacuum. For the dimming referred to above this relativistic time dilation leads to further reduced numbers of received photons per time unit compared to the number of radiated photons. Combining this time dilation effect with formula (5) we obtain formula (8) 


$$
N / N_{0}=\frac{\sqrt{1-\frac{V^{2}}{c^{2}}}}{1+\frac{V}{c}}
$$

This resemblance to the Doppler effect formula (1) is clear, because both describe fundamentally the same effect. Essentially the classical Doppler effect formula describes the wave component of light and formula (8) describes the corpuscular component of light.

Taking the relativistic effects into account we obtain the dimming effect for an object moving away from the observer with the speed $0.1 c$ as

$$
N / N_{0}=\frac{\sqrt{1-0.1 * 0.1}}{1+0.1}=0.904
$$

and for an object moving away from the observer with the speed $0.5 c$ as

$$
N / N_{0}=\frac{\sqrt{1-0.5 * 0.5}}{1+0.5} \quad=0.577
$$

In addition to Special Relativity, "Relativistic effects" covers gravitational time dilation as described by General Relativity and also by cosmological expansion. Therefore, we can expect that anywhere that the Doppler effect applies for waves (formula 1) the dimming effect will also take place (formula 8).

If confirmed by experiment the described dimming effect must be taken into account for astronomical calculations involving the "Standard Candle" and in particular the "Supernova Cosmology Project" [3], which has claimed the acceleration of the Universe's expansion and led to the introduction of dark energy.

\section{References}

1. Landau L. D., Lifshits E. M. Teoriia polia. - Izdanie 7-e, ispravlennoe. [Theory of the field. - 7th edition, revised] — M.: Nauka, 1988. — Pp. 158-159. — (Teoreticheskaia fizika, tom II ["Theoretical Physics", volume II]). — ISBN 5-02-014420-7.

2. Cotăescu Ion I., "Longitudinal Doppler effect in de Sitter relativity”, arXiv: 2007.13590, 2020

3. Goldhaber, Gerson (2009). "The Acceleration of the Expansion of the Universe: A Brief Early History of the Supernova Cosmology Project (SCP)". AIP Conference Proceedings. 1166: 53. arXiv:0907.3526. Bibcode:2009AIPC.1166...53G. doi:10.1063/1.3232196. 